\title{
Artigo Orignal
}

\section{EVOLUÇÃO ANTROPOMÉTRICA E SINTOMAS GASTROINTESTINAIS EM PACIENTES QUE RECEBERAM SUPLEMENTOS NUTRICIONAIS OU NUTRIÇÃO ENTERAL}

Juliana Maria Faccioli Sicchieri*1, Maria do Rosário del Lama de Unamuno², Júlio Sérgio Marchini³ ${ }^{3}$, Selma Freire de Carvalho da Cunha ${ }^{4}$

Trabalho realizado na Faculdade de Medicina de Ribeirão Preto - USP - Disciplina de Nutrologia do Departamento de Clínica, Ribeirão Preto, SP

*Correspondência:

Av. dos Bandeirantes, 3900

Ribeirão Preto-SP

CEP $14048-900$

\begin{abstract}
RESUMO
Овјетіvo. Em pacientes hospitalizados, comparar a evolução de variáveis antropométricas e a ocorrência de efeitos adversos relacionados ao consumo de suplementos nutricionais e infusão de nutrição enteral.

MÉTodos. 10 pacientes que recebiam suplementos nutricionais (Grupo SN) e 20 em nutrição enteral (Grupo NE), pareados para o gênero, idade ( $50 \pm 21$ vs $49 \pm 23$ anos) e afecções de base foram submetidos à avaliação antropométrica ao início e término da terapêutica. Diariamente, foi aplicado um questionário semi-estruturado referente às queixas gastrointestinais. Determinaram-se as diferenças estatísticas obtidas entre o início e o final da terapêutica (teste t para amostras dependentes) e entre os grupos de estudo (teste t para amostras independentes).

Resultados. A circunferência muscular do braço aumentou nos pacientes do Grupo NE (80 \pm 15 vs $85 \pm 15 \%$ de adequação, $p=0,009)$ e diminuiu no Grupo SN ( $96 \pm 14$ vs $92 \pm 14 \%$ de adequação, $p=0,04)$. Náuseas e vômitos foram mais frequentes no Grupo SN (60 vs $10 \%, p=0,01)$ e as queixas relacionadas ao sabor dos produtos ocorreram em $30 \%$ dos casos.

Conclusão. Os pacientes que receberam suplementos nutricionais apresentaram maior ocorrência de queixas gastrointestinais e evolução desfavorável dos parâmetros antropométricos. Os dados obtidos no estudo não evidenciam o benefício da suplementação nutricional de rotina em pacientes hospitalizados.
\end{abstract}

UnItermos: Terapia nutricional. Suplemento nutricional. Antropometria. Sintomas gastrointestinais.

\section{INTRODUÇÃO}

Qualidade em terapia nutricional representa a condição de redução do custo e do tempo de permanência do paciente no ambiente hospitalar, que implica em evolução clínica favorável, melhora de diversos parâmetros de qualidade de vida ${ }^{1,2}$ e na redução da taxa de morbidade e mortalidade. 0 fornecimento adequado de nutrientes mantém ou recupera o estado nutricional, atenua os efeitos adversos da resposta metabólica às lesões de natureza diversas e minimiza o acometimento da estrutura e funcionamento de órgãos vitais ${ }^{3}$. Mesmo que os produtos utilizados em terapia nutricional tenham composição adequada, o preparo dos profissionais de saúde na indicação e seleção da modalidade de terapia nutricional contribui para a qualidade da assistência prestada aos pacientes hospitalizados.

Pacientes com apetite preservado e trato gastrointestinal íntegro beneficiam-se com a terapia oral. $\mathrm{Na}$ maioria das situações clínicas, é possível atingir as necessidades nutricionais pela modificação na consistência dos alimentos e/ou com a ingestão dos suplementos nutricionais ${ }^{2}$, oferecidos entre as refeições principais. Deficiências nutricionais prévias, comuns entre indivíduos hospitalizados, têm justificado o emprego de suplementos ${ }^{4}$, com o objetivo de suprir a ingestão oral insuficiente ${ }^{5}$. A colaboração efetiva dos pacientes é essencial para garantir que o volume prescrito dos suplementos nutricionais seja adequadamente ingerido. A hiporexia, a saciedade precoce, a presença de náuseas e as desordens neurológicas que dificultam a deglutição ${ }^{6}$ são fatores que influenciam na aceitação dos suplementos nutricionais e interferem no sucesso da terapia. Além disso, o significado emocional atribuído à alimentação, a perda da autonomia quanto às preferências e as mudanças no padrão alimentar podem ser fatores de conflito que geram sentimentos de raiva, ansiedade e depressão e interferem na aceitação $0^{7,8}$.

Embora os suplementos nutricionais sejam largamente utilizados no ambiente hospitalar, na maioria das vezes não há informações adequadas do estado nutricional antes do início da

1. Nutricionista da Divisão de Nutrologia do Departamento de Clínica Médica da Faculdade de Medicina de Ribeirão Preto-US

2. Especialista em nutrição - Enfermeira chefe da Unidade Metabólica, disciplina de Nutrologia da Faculdade de Medicina de Ribeirão Preto-USP

3. Professor titular da Divisão de Nutrologia do Departamento de Clínica Médica da Faculdade de Medicina de Ribeirão Preto-USP

4. Professor Doutor do Curso de Nutrição e Metabolismo da Faculdade de Medicina de Ribeirão Preto-USP 
terapia, o que inviabiliza a análise da adequação da prescrição ${ }^{9}$. Além disso, faltam protocolos para o emprego racional dos suplementos, podendo determinar a hipervalorização desta modalidade terapêutica. Considerando-se a decisão técnica da equipe multidisciplinar, os aspectos éticos e o desejo do paciente e/ou seus familiares, se após 5 a 7 dias não houver melhora nos parâmetros objetivos da avaliação, está indicada a terapia de nutrição enteral ${ }^{10}$, cuja eficácia tem sido bem documentada ${ }^{11}$. 0 objetivo do presente estudo foi comparar a evolução dos parâmetros antropométricos e dos efeitos adversos relacionados à ingestão de suplementos nutricionais e à nutrição enteral em pacientes hospitalizados.

\section{Métodos}

0 estudo foi conduzido com 30 pacientes adultos (idade > 21 anos), de ambos os gêneros, internados nas diversas enfermarias de Clínica Médica do Hospital das Clínicas da Faculdade de Medicina de Ribeirão Preto - USP, após aprovação pelo Comitê de Ética em Pesquisa da Instituição (Processo $9017 / 2007)$. Foram selecionados aleatoriamente os pacientes que recebiam dieta por via oral, acrescida de suplementos nutricionais (Grupo SN, $n=10$ ) ou de nutrição enteral (Grupo $\mathrm{NE}, \mathrm{n}=20$ ) por um período mínimo de cinco dias e que concordassem com a participação voluntária na pesquisa. Foram excluídos os pacientes com comprometimento da capacidade cognitiva ou de verbalização, como os sedados, disfásicos, inconscientes ou confusos, além daqueles sem condições de submeter-se à avaliação antropométrica.

Os pesquisadores não tiveram qualquer interferência na indicação, na determinação do volume prescrito ou na interrupção dos suplementos nutricionais e da nutrição enteral, que foram feitos pelos médicos responsáveis, conforme protocolo de rotina assistencial do serviço. Todos os participantes do estudo recebiam o mesmo produto industrializado, em pó, diluído em água, na concentração de 1,0 ou $1,5 \mathrm{kcal} / \mathrm{mL}$, que foram oferecidos por via oral (como suplemento nutricional) ou por sonda nasoentérica (como nutrição enteral).

No prontuário, foram coletados os dados de identificação, que incluíram a idade e os diagnósticos principais. Semanalmente, foi realizada avaliação antropométrica que consistiu na medida do peso corporal, do comprimento e da circunferência braquial e da prega cutânea tricipital. 0 peso corporal foi aferido em balança digital FilizolaR , com precisão de 100 gramas, estando o indivíduo de pé, descalço e com o mínimo de roupa. O comprimento do braço (CB) foi determinado com fita métrica inextensível no braço não dominante, pela distância entre olecrano e acrômio. No ponto médio, foi aferida a circunferência do braço (CB, em centímetro) e a prega cutânea tricipital (PCT, em milímetros), usando-se um plicômetro Lange Skinfoldr, sendo considerado o valor médio de três aferições consecutivas. A circunferência muscular do braço foi determinada por equação $(\mathrm{CM}=\mathrm{CB}-3,14216 \times \mathrm{PT}$, em cm). Os dados antropométricos, expressos em porcentagem dos valores ideais, foram obtidos por apenas um avaliador previamente treinado com as técnicas padronizadas ${ }^{12}$.

Diariamente, foi aplicado um questionário semiestruturado com questões referentes à sensação subjetiva de melhora do estado geral, queixas gastrointestinais, desconforto e insatisfação com a auto-imagem relacionados com a presença da sonda nasoentérica. 0 estudo não incluiu a avaliação quantitativa da oferta energética e proteica e os voluntários foram questionados quanto aos motivos da recusa do suplemento nutricional e as sugestões que pudessem melhorar sua aceitação.

Os dados obtidos foram tabulados em banco de dados do EXCEL e a análise estatística feita no programa computadorizado STATISTICS 6.0. O teste de Shapiro-Wilks foi aplicado para testar a normalidade da distribuição dos dados obtidos e o teste de Levine avaliou a homogeneidade das variâncias. A comparação entre os diversos parâmetros entre os pacientes que receberam terapia nutricional enteral ou suplementos orais foi feita pelo teste $T$ de Student para amostras independentes e variâncias homogênas. Os resultados obtidos no início e final da terapêutica nutricional foram comparados pelo teste T de Student para amostras dependentes. Diferenças entre proporções foram determinadas pelo teste do Qui quadrado de Yates ou teste exato de Fisher, dependendo da frequência das variáveis categóricas. Foram consideradas diferenças significativas quando $p \leq 0,05$.

\section{Resultados}

Não houve diferença na porcentagem de indivíduos do gênero feminino $(60 \%$ vs. $40 \%)$ e na idade $(49,9 \pm 20,8$ vs 49,0 $\pm 22,5$ anos) dos pacientes receberam suplementos nutricionais ou nutrição enteral. Os indivíduos foram pareados quanto à afecção de base, que foram agrupadas em doenças cardíacas ( $20 \%$ vs. $10 \%)$, neuropsiquiátricas (20\% vs. $35 \%)$, neoplásicas ( $20 \%$ vs. $20 \%$ ), do trato gastrointestinal (30\% vs. $15 \%$ ) e endocrinológicas ( $10 \%$ vs. $20 \%$ ), respectivamente para o Grupo SN e NE.

Embora não tenha havido diferença significativa $(p=0,08)$, os suplementos nutricionais (13,5 dias, variando de 5 a 28 ) foram oferecidos por menor período que a nutrição enteral $(23,5$ dias, variando de 7-125). Neste período, os pacientes que receberam os suplementos nutricionais apresentaram redução de $3,6 \mathrm{~kg}$ no peso corporal, variando entre a perda de $10 \mathrm{~kg}$ até 0 ganho de $1 \mathrm{~kg}$. No Grupo NE, houve ganho de 1,3 kg, variando entre a perda de $3 \mathrm{~kg}$ ao acréscimo de $18,5 \mathrm{~kg}$. A evolução dos demais dados antropométricos é mostrada na Tabela 1. No início da terapia nutricional, a CB e CM dos pacientes do Grupo SN eram maiores que no Grupo NE. Entretanto, ao final da terapêutica, houve piora dos parâmetros antropométricos no Grupo SN enquanto os pacientes do Grupo NE melhoraram estes parâmetros.

A análise dos dados obtidos no questionário semiestruturado mostrou que a sensação de melhora subjetiva foi citada apenas por dois pacientes $(10 \%)$ que recebiam nutrição enteral. Queixas relacionadas ao sabor dos suplementos nutricionais ocorreram em $30 \%$ dos pacientes. Para melhorar a aceitação, os pacientes sugeriram que os suplementos fossem oferecidos em horários distantes das refeições principais, com sabores variados, em menor temperatura e volume por porcionamento. Entre os que recebiam nutrição enteral, houve baixa frequência de desconforto nasal $(3,33 \%)$ e insatisfação com a auto-imagem $(3,33 \%)$ relacionadas com presença sonda. A Tabela 2 expressa a frequência de sinais e sintomas 


\begin{abstract}
Tabela 1 - Valores de circunferência do braço (CB), prega cutânea tricipital (PCT) e circunferência muscular do braço (CM), expressos em porcentagem de adequação dos valores de normalidade, obtidos no início e no final do estudo dos pacientes que receberam suplementos nutricionais (Grupo SN) ou nutrição enteral (Grupo NE)
\end{abstract}

\begin{tabular}{lccccc}
\hline & \multicolumn{2}{c}{ Inicial } & & \multicolumn{2}{c}{ Final } \\
& Grupo SN & Grupo NE & & Grupo SN & Grupo NE \\
\cline { 2 - 3 } \cline { 5 - 6 } & $(n=10)$ & $(n=20)$ & & $(n=10)$ & $(n=20)$ \\
\hline CB $^{a, b, c}$ & $96,04 \pm 15,93$ & $81,02 \pm 15,32$ & & $90,70 \pm 15,61$ & $85,76 \pm 15,39$ \\
PCT $^{b}$ & $101,50 \pm 30,63$ & $86,90 \pm 27,69$ & & $85,56 \pm 26,99$ & $91,84 \pm 32,19$ \\
CM $^{a}, b, c$ & $95,56 \pm 13,75$ & $80,52 \pm 14,67$ & & $92,15 \pm 14,34$ & $85,01 \pm 15,32$ \\
\hline (a) $=$ no momento inicial, existe diferença estatística entre os Grupos SN e NE \\
(b) = No Grupo SN, existe diferença entre os momentos da avaliação \\
(c) = No Grupo NE, existe diferença entre os momentos da avaliação
\end{tabular}

Tabela 2 - Valores percentuais e absolutos das queixas, sinais e sintomas de pacientes que receberam suplementos nutricionais (Grupo SN) ou nutrição enteral (Grupo NE)

\begin{tabular}{lccc}
\hline & $\begin{array}{c}\text { Grupo SN } \\
\text { (n= 10) }\end{array}$ & $\begin{array}{c}\text { Grupo NE } \\
\text { (n= 20) }\end{array}$ & p valor \\
\hline Hiporexia & $30 \%(3)$ & $5 \%(1)$ & 0,18 \\
Náuseas ou vômitos & $60 \%(6)$ & $10 \%(2)$ & 0,01 \\
Dor abdominal em cólica & $10 \%(1)$ & $30 \%(6)$ & 0,19 \\
Diarréia & $10 \%(1)$ & $45 \%(9)$ & 0,13 \\
Distensão abdominal & $20 \%(2)$ & $20 \%(4)$ & 1,0 \\
Flatulência & 0 & $5 \%(1)$ & 0,67 \\
\hline
\end{tabular}

relacionados à terapia nutricional, na qual as queixas de náuseas e vômitos foram mais frequentes entre os pacientes do Grupo SN.

\section{Discussão}

Comparados com os pacientes que receberam nutrição enteral, aqueles que ingeriam suplementos nutricionais apresentaram piora nos parâmetros antropométricos e maior ocorrência de náuseas ou vômitos. O peso corporal pode sofrer interferências do estado de hidratação de forma que, em muitas ocasiões, a evolução ponderal constitui-se num parâmetro pouco confiável de avaliação nutricional. Embora haja fatores de erro relacionados à seleção de equipamentos, emprego de técnicas não padronizadas e variação entre avaliadores, as medidas antropométricas do braço constituem-se medida alternativa em tais situações ${ }^{13}$. Mesmo considerando os maiores valores das circunferências do braço e muscular ao início do estudo, os pacientes que receberam suplementos nutricionais apresentaram evolução antropométrica desfavorável quando comparada com aqueles que receberam nutrição enteral.

Alguns estudos avaliam a evolução de pacientes ingerindo suplementos com diferentes concentrações energéticas ( 1,0 ou $2,0 \mathrm{kcal} / \mathrm{mL})^{14}$ e com variação nas fontes nutricionais, provenientes de alimentos regulares (cereais, produtos lácteos e ovoalbumina) ou a partir de produtos industriais prontos para uso ${ }^{15}$. $\mathrm{Na}$ maioria dos estudos da literatura, os indivíduos do grupo controle não receberam qualquer intervenção dietética ou ingerem placebo, o que representa grande diferença metodológica em relação ao presente estudo, que compara os resultados obtidos em duas diferentes modalidades de terapia nutricional.

Na literatura não há uniformidade na condição clínica dos indivíduos que recebem suplementos nutricionais na duração da intervenção e nos critérios utilizados para avaliar a eficácia, entre eles, a melhora subjetiva, as mudanças nos parâmetros antropométricos, a ocorrência dos efeitos adversos, a taxa de morbidade e mortalidade ${ }^{16}$. Quando os suplementos foram ingeridos por períodos prolongados, idosos da comunidade apresentaram melhora do quadro clínico e diminuição da morbidade e mortalidade ${ }^{17}$. Os suplementos nutricionais orais também melhoraram o estado nutricional de pacientes em risco ou com subnutrição definida ${ }^{18}$, em portadores cirrose alcoóli$\mathrm{Ca}^{19}$, insuficiência renal crônica em tratamento hemodialítico ${ }^{20}$ ou sob diálise peritoneal ${ }^{21}$.

A suplementação nutricional não modificou a concentração plasmática dos marcadores nutricionais de idosos hospitalizados com úlcera de pressão ${ }^{4}$ ou a mortalidade daqueles com fratura de quadril, apesar de reduzir a frequência de complicações ${ }^{22}$. Em metanálise incluindo 55 estudos não foram mostradas evidências de benefícios do uso de suplementos industrializados ou de fontes alimentares fortificadas em pessoas como estado nutricional preservado, embora os idosos subnutridos tenham apresentado melhora dos parâmetros antropométricos, redução das complicações e da mortalidade ${ }^{16}$.

Em idosos ${ }^{23}$ e indivíduos infectados por HIV15, a baixa aceitação de suplementos nutricionais foi associada à intolerância ao produ to e à ocorrência de náuseas, vômitos e diarreia. $\mathrm{Na}$ maioria dos estudos, a ocorrência dos potenciais efeitos adversos decorrentes da ingestão dos suplementos nutricionais não é avaliada ${ }^{5}$ ou, quando citada, não há comparação com grupo controle e os resultados são discordantes ${ }^{3}$. Comparados com 0 grupo controle, os indivíduos que receberam suplementos nutricionais apresentaram maior ocorrência de náuseas, vômitos e diarréia $24,25,26$.

No presente estudo, a evolução desfavorável dos pacientes que receberam suplementos nutricionais não pode ser atribuída às condições clínicas relacionadas à doença de base, aos fatores de erro decorrentes da avaliação antropométrica ou à qualidade nutricional da formulação utilizada. Embora não tenhamos informações sobre o consumo energético e proteico durante o estudo, é provável que a piora dos parâmetros antropométricos observada nos indivíduos que receberam suplementos orais possa ser atribuída à ingestão nutricional insuficiente. Tais resultados podem estar relacionados à gravidade dos casos e condicionados à baixa palatabilidade, atratividade e monotonia de sabor dos produtos, à desconsideração sobre hábitos alimentares dos pacientes e à ocorrência de queixas gastrointestinais.

É notável a tentativa de manter a via oral, mas é provável que tenha havido relutância em iniciar a nutrição enteral, considerando que os suplementos nutricionais se mostraram ineficazes em manter ou melhorar o estado nutricional de pacientes hosp italizados. Mesmo cientes das necessidades nutricionais básicas, a equipe de saúde pode supervalorizar as complicações da nutrição enteral e projetar sentimentos negativos diante de paciente em privados da alimentação por via oral ${ }^{27,28}$. 
SICCHIERI J MF ET AL.

Embora a suplementação nutricional possa beneficiar muitos indivíduos, os dados obtidos no presente estudo não evidenciam o benefício da suplementação nutricional de rotina para pacientes hospitalizados. A elaboração e implantação de protocolos específicos para o uso de suplementos nutricionais pode otimizar os resultados obtidos com esta modalidade terapêutica ${ }^{7}$. O acompanhamento diário do nutricionista é essencial na montagem de estratégias para melhorar a aceitação dos suplementos nutricionais, na monitorização da terapia nutricional e na proposta de mudança da modalidade terapêutica em pacientes hospitalizados.

\section{Conflito de interesse: não há}

\section{SUMMARY}

Anthropometric eVolution and Gastrointestinal COMplaints in ORAL NUTRITIONAL SUPPLEMENTATION AND ENTERAL NUTRITIONAL THERAPY

Oвjective. To compare the evolution of anthropometric variables and the occurrence of adverse effects related to the ingestion of nutritional food supplements and enteral diet administration in hospitalized patients.

Methods. The study was performed in the Clinical Medicine wards of the Hospital of the School of Medicine of Ribeirão Preto, USP, with 10 patients receiving nutrition supplements, and 20 patients under enteral nutrition therapy, paired by gender, age ( $50 \pm 21$ vs $49 \pm 23$ years) and basic afflictions. All were volunteers submitted to anthropometric evaluation at the beginning and end of nutritional therapy, utilizing standard techniques. A semi-structured questionnaire was applied daily referring to gastrointestinal complaints. Statistical differences between onset and final results (t-test for dependent samples) and between-group differences (t-test for independent samples) were estimated.

RESULTS. Arm muscle circumference increased in patients receiving enteral nutrition therapy (80 \pm 15 vs $85 \pm 15 \%$ adequacy, $p=0.009)$ and decreased in those receiving nutritional supplements ( $96 \pm 14$ vs $92 \pm 14 \%$ adequacy, $p$ $=0.04)$. Nausea and vomiting were more frequent in the latter ( 60 vs $10 \%, p=0.01$ ); complaints about taste of the products were reported by $30 \%$ of the cases.

Conclusion. Patients who received oral nutrition supplements reported gastrointestinal complaints and had a less favorable anthropometric evolution. Study data did not disclose the benefit of routine nutrition supplements for hospitalized patients. [Rev Assoc Med Bras 2009; 55(2): 149-52 ]

KEY WORDS: Nutritional therapy, oral nutritional supplementation, anthropometry, gastrointestinal symptoms.

\section{ReferênCIAS}

1. Corral FP, Abraira V. Autoperception and satisfaction with health: two medical care markers in elderly hospitalized patients. Quality of life as an outcome estimate of clinical practice. J Clin Epidemiol. 1995;48:1031-40.

2. Ottery FD. Supportive nutrition to prevent cachexia and improve quality of live. Semin Oncol. 1995; 22:98-111.

3. Gallagher-Allred CR, Voss AC, Finn SC, McCamish MA. Malnutrition and clinical outcomes: the case for medical nutrition therapy. J Am Diet Assoc. 1996;96:361-6.
4. Raffoul W, Far MS, Cayeux MC, Berger M M. Nutritional status and food intake in nine patients with chronic low-limb ulcers and pressure ulcers: importance of oral supplements. Nutrition. 2006;22:82-8.

5. Shueren Van Bokhorst-de van der MA. Nutritional support strategies for malnourished cancer patients. Eur J Oncol Nurs. 2005;9:S74-83.

6. Hessov I. Oral diet administration and supplementation. In: Payne-J ames I, Grimble G, Silk D. Artificial nutrition support in clinical practice. London: Edward Arnold; 1995..p.187-96.

7. Boog MCF. Dificuldades encontradas por médicos e enfermeiros na abordagem de problemas alimentares. Rev Nutr. 1999:12:261-72.

8. Barbosa J AG, Freitas MIF. Representações sociais sobre a alimentação por sonda obtidas de pacientes adultos hospitalizados. Rev Latinoam Enferm. 2005;13:235-42.

9. Gale CR, Edington J, Coles SJ, Martyn CN. Patterns of prescribing of nutritional supplements in the United Kingdom. Clin Nutr. 2001;20:333-7.

10. Stroud $M$, Duncan $H$, Nightingale J. Guidelines for enteral feeding in adult hospital patients. Gut. 2003;52(Suppl 7):1-12

11. Cunha, SFC, Sicchieri, JMF, Unamuno, MRDL, Borges, NJBG, Marchini, J S. Terapia de nutrição enteral. In: Vannucchi, H, Marchini, J S, editores. Nutrição clínica. Rio de Janeiro: Guanabara Koogan; 2007. p.78-95.

12. Rombeau J L, Caldwell MD, Forlaw L, Guenter PA. Atlas of nutritional support techiniques. Boston: Bittle Brown; 1989.

13. WHO Expert Committee on Physical Status: the use and the interpretation of antropometry. Switzerland: WHO; 1995. [WHO Technical series n.854]

14. Collins CE, Kershaw J, Brockington S. Effect of nutritional supplements on wound healing in home-nursed elderly: a randomized trial. Nutrition. 2005:21:147-155

15. Charlin V, Carrasco F, Sepulveda C, Torres M, Kehr J. Nutritional supplementation according to energy and protein requirements in malnourished HIV-infected patients. Arch Latinoam Nutr. 2002:52:267-73.

16. Caballero YE, Atalah ES. Evaluación De La Aceptabilidad Y Consumo de un suplemento alimentario en La República de Panama. Rev Chil Nutr. 2003;30:133-6.

17. Fiatarone MA, O'N eill EF, Ryan ND, Clements KM, Solares GR, Nelson ME, et al. Exercise training and nutritional supplementation for physical frailty in very elderly people. N Engl J Med. 1994;330:1769-75.

18. Gazzotti C, Arnaud-Battandier F, Parello M, Farine S, Seidel L, Albert A, et al. Prevention of malnutrition in older people during and after hospitalisation: results from a randomised controlled clinical trial. Age Ageing. 2003;32:321-5.

19. Vermeeren MA, Wouters EF, Geraerts-Keeris AJ, Schols AM. Nutritional support in patients with chronic obstructive pulmonary disease during hospitalization for an acute exacerbation; a randomized controlled feasibility trial. Clin Nutr. 2004;23:1184-92.

20. Milne AC, Avenell A, Potter J. Meta-analysis: protein and energy supplementation in older people. Ann Intern Med. 2006;144:37-48.

21. Avenell A, Handoll $\mathrm{HH}$. N utritional supplementation for hip fracture aftercare in older people. Cochrane Database Syst Rev 2005;(2) CD001880. Available from: http://www.ncbi.nlm.nih.gov.

22. Potter J M. Oral supplements in the elderly. Curr Opin Clin Nutr Metab Care. 2001;4:21-8.

23. Lauque S, Arnaud-Battandier F, Mansourian R, Guigoz Y, Paintin M, Nourhashemi $F$, et al. Protein-energy oral supplementation in malnourished nursing-home residents. A controlled trial. Age Ageing. 2000;29:51-6.

24. Cunha L, Happi Nono M, Guibert AL, Nidegger D, Beau P, Beauchant $M$. Effects of prolonged oral nutritional support in malnourished cirrhotic patients: results of a pilot study. Gastroenterol Clin Biol. 2004;28:36-9.

25. Caglar K, Fedje L, Dimmitt R, Hakim RM, Shyr Y, Ikizler TA Therapeutic effects of oral nutritional supplementation during hemodialysis. Kidney Int. 2002;62:1054-9.

26. Boudville N, Rangan A, Moody H. Oral nutritional supplementation increases caloric and protein intake in peritoneal dialysis patients. Am J Kidney Dis. 2003;41:658-63.

27. Madigan SM, Fleming P, McCann S, Wright ME, MacAuley D. General practitioners involvement in enteral tube feeding at home: a qualitative study. BMC Fam Pract. 2007;15:8-29.

28. Winkler MF. Food for thought: its more than nutrition. JPEN J Parenter Enteral Nutr. 2007;31:334-40.

Artigo recebido: 05/12/07

Aceito para publicação: 13/09/08 